\title{
Melt Electrowriting of Poly(vinylidene fluoride-co-trifluoroethylene)
}

Juliane C. Kade ${ }^{1}$, Biranche Tandon ${ }^{1}$, Jan Weichhold ${ }^{1}$, Dario Pisignano ${ }^{2,3}$, Luana Persano ${ }^{2}$, Robert Luxenhofer ${ }^{4,5^{*}}$, Paul D. Dalton ${ }^{1 *}$

* Corresponding authors

${ }^{1}$ Department of Functional Materials in Medicine and Dentistry and Bavarian Polymer Institute University Hospital Würzburg, Pleicherwall 2, 97070 Würzburg, Germany

${ }^{2}$ NEST, Istituto Nanoscienze-CNR and Scuola Normale Superiore, Piazza San Silvestro 12, I-56127 Pisa, Italy

${ }^{3}$ Dipartimento di Fisica, Università di Pisa, Largo B. Pontecorvo 3, I-56127 Pisa, Italy

${ }^{4}$ Polymer Functional Materials, Chair for Advanced Materials Synthesis, Department of Chemistry and Pharmacy, Julius-Maximilians-University Würzburg, Würzburg, Germany.

${ }^{5}$ Soft Matter Chemistry, Department Chemistry, and Helsinki Institute of Sustainability Science, Faculty of Science University of Helsinki, 00014 Helsinki, Finland

Correspondence to:

Prof. Dr. Robert Luxenhofer: robert.luxenhofer@helsinki.fi

Prof. Dr. Paul D. Dalton: paul.dalton@fmz.uni-wuerzburg.de 


\begin{abstract}
Poly(vinylidene fluoride-co-trifluoroethylene) (PVDF-TrFE) is an electroactive polymer with growing interest for applications in biomedical materials and flexible electronics. In this study, a solvent-free additive manufacturing technique called melt electrowriting (MEW) has been utilized to fabricate well-defined microperiodic structures of the copolymer (P(VDF-co-TrFE)). Melt electrowriting of the highly viscous polymer melt was initiated using a heated collector at temperatures above $120^{\circ} \mathrm{C}$ and required remarkably slow collector speeds below $100 \mathrm{~mm} / \mathrm{min}$. The fiber surface morphology was affected by the collector speed and an increase in $\beta$-phase was observed for scaffolds when compared to the unprocessed powder. Videography shows vibrations of the (P(VDF-co-TrFE)) jet previously unseen during melt electrowriting, likely due to repeated charge buildup and discharge. Furthermore, piezo-force microscopy measurements demonstrated the electroactive properties of MEW-fabricated fibers. This research therefore achieves the melt electrohydrodynamic processing of fibers with micrometer resolution into defined structures with an important electroactive polymer.
\end{abstract}




\section{Introduction}

Electroactive polymers with piezoelectric properties are of increasing interest for biomedical applications, in particular for the electrical stimulation of cells without the need for an external power supply [1, 2]. Poly(vinylidene fluoride) (PVDF) and the copolymer poly(vinylidene fluoride-co-trifluoroethylene) ( $\mathrm{P}(\mathrm{VDF}-\mathrm{co}-\mathrm{TrFE}))$ are often used for biomedical applications [2-6] owing to their flexibility, non-toxicity and good chemical resistance $[1,2,4$, 7]. The piezoelectric properties of such polymers emerge when the crystalline structure is in the all-trans conformation which results in a net dipole moment [1]. PVDF is piezoelectric in the $\beta$-phase conformation, the content of which can be increased at the expense of the $\alpha$-phase (trans-gauche-trans-gauche confirmation) by stretching, poling or annealing of the raw material [8]. In contrast, $\mathrm{P}(\mathrm{VDF}-\mathrm{co}-\mathrm{TrFE})$ inherently prefers to crystallize into a crystal structure similar to the all-trans conformation ( $\beta$-phase) due to the steric hindrance provided by the higher amount of fluorine atoms introduced by $\operatorname{TrFE}[1,9-11]$. However, to improve the alignment of polymer chains/crystalline domains and generate a continuous macroscopic polarization, the copolymer $\mathrm{P}(\mathrm{VDF}-\mathrm{co}-\mathrm{TrFE})$ needs to be polarized by an electric field [11].

Solution electrospun PVDF and P(VDF-co-TrFE) have been extensively studied for tissue engineering applications by Arinzeh and colleagues [5, 12-16]. Small diameter P(VDF-coTrFE) fibers $(\sim 970 \pm 480 \mathrm{~nm})$ showed in vitro cytocompatibility using human skin fibroblasts [16] and improved neurite extension using Schwann cells and/or dorsal root ganglion $[5,15]$ and human neural stem / progenitor cells [14]. An increase in chondrogenic and/or osteogenic differentiation of human mesenchymal stem cells on PVDF [13] and P(VDF-co-TrFE) fibers [12] has also been shown.

An alternative and solvent-free processing approach for fabricating fibers is melt electrospinning. When accurate fiber placement is desired, one can utilize melt electrowriting (MEW) which gives excellent control over fiber placement. The enhanced control provided by MEW allows to direct-write scaffolds with defined structures and pore sizes [17-21]. Previously, 
a piezoelectric polymer (PVDF) was processed via MEW for the first time, with the fibers having increased $\beta$-phase content compared to the unprocessed powder [22]. The copolymer $\mathrm{P}(\mathrm{VDF}-\mathrm{co}-\mathrm{TrFE})$ has, to date, been processed predominantly using solution electrospinning (SES) and film drawing. Both approaches require post-treatment to either remove toxic solvents, a particularly pertinent step for biomedical applications, or to maximize the macroscopic polarization ( $\beta$-phase) by applying an electrical field [11].

This study investigates processing of $\mathrm{P}(\mathrm{VDF}-\mathrm{co}-\mathrm{TrFE})$ using MEW and determines the printability of the copolymer with this technique. The influence of the high viscosity of the material on the MEW-process and the resulting scaffolds is inquired. Furthermore, a heated collector was essential with the temperature found to affect the overall crystallinity and $\beta$-phase content in the fibers. A collector temperature of $120^{\circ} \mathrm{C}$, close to the Curie temperature of the polymer when heating, was found to improve crystallization and consequently led to higher amount of $\beta$-phase. Interestingly, buckling of the fibers occurred when the collector temperature was reduced to room temperature.

\section{Experimental Section}

\subsection{Materials}

P(VDF-co-TrFE) (Solvene® 200/P200; \#900895) was purchased from Sigma Aldrich and used as received. The melt flow index (MFI), as provided by the manufacturer, is $25 \mathrm{~g} / 10 \mathrm{~min}$ (ASTM D1238).

\subsection{Melt electrowriting (MEW)}

The MEW processing of $\mathrm{P}(\mathrm{VDF}-\mathrm{co}-\mathrm{TrFE})$ was performed with a custom-built device as previously described [23], which operated at a nitrogen $\left(\mathrm{N}_{2}\right)$ pressure of 0.5 bar. A stainlesssteel nozzle was attached to a $3 \mathrm{~mL}$ glass syringe (Fortuna Optima $3 \mathrm{~mL}$ Luer Lock). The flattipped nozzle was prepared by grinding an injection cannula (22-gauge with Luer Lock, Carl 
Roth, Germany) to a length of $7.0 \pm 0.2 \mathrm{~mm}$. The print head and nozzle tip temperatures were set to a value of $170 \pm 2^{\circ} \mathrm{C}$ as reported by respective thermocouples. A potential difference of $+3.70 \pm 0.20 \mathrm{kV}$ was applied between the nozzle and the grounded collector plate. MEW was maintained at a collector distance of $4.4 \pm 0.5 \mathrm{~mm}$, with $3.0 \pm 0.2 \mathrm{~mm}$ of the nozzle protruding beyond the electrowriting head. A heated collector was custom-built as previously described [23] and set to $120-135 \pm 5^{\circ} \mathrm{C}$ during printing. Glass microscope slides (VWR, ground edge, cat \# 631-1550) were chosen as a printing substrate and were placed on top of the heated collector. The glass syringe was loaded with approximately $0.5 \mathrm{~g}$ of $\mathrm{P}(\mathrm{VDF}-\mathrm{co}-\mathrm{TrFE})$ and preheated for at least 30 min prior to printing.

\section{$2.3 X$-ray diffraction $(X R D)$}

XRD spectra were collected using a BRUKER D8 ADVANCE. The operating voltage and current used were $30 \mathrm{kV}$ and $53.3 \mathrm{~mA}$. The samples were irradiated with $\mathrm{Cu} \mathrm{K} \alpha$ radiation using a step size of $0.015^{\circ}$, a dwell time of $0.7 \mathrm{~s}$ and a rotation of $15 \mathrm{rpm}$ in the $2 \theta$ range of $10^{\circ}$ to $80^{\circ}$ with a zero-background sample holder made of a silicon single crystal. The raw powder and MEW processed scaffolds printed with different speeds at two different collector temperatures $\left(120\right.$ and $\left.135^{\circ} \mathrm{C}\right)$ were measured. Background corrections on the measurements were done using a beam knife and the software DIFFRAC.EVA.

\subsection{Imaging and videography}

Stereomicroscope images were taken with a Discovery V20 (Carl Zeiss Microscopy GmbH, Germany). SEM imaging of the MEW-processed fibers and scaffolds was performed with a Crossbeam 340 (Carl Zeiss Microscopy GmbH, Germany) instrument with all samples sputtercoated with approximately $3 \mathrm{~nm}$ of platinum (Leica EM ACE600). Videography was made using a Sony Alpha 7 and Nikon Z6 digital camera with Nikon ED 200 mm lens. Editing of the videos was performed using the software DaVinci Resolve 16.2.7.01. 


\subsubsection{Fiber diameter}

A tabletop SEM Microscope (TM3030p, Hitachi High-Tech Corporation) was used to take images from three different samples with four printed lines per collection speed. These images were then used to measure the diameter with ImageJ (Version 1.52a, NIH, USA) at around 50 different locations on the fiber per collection speed.

\subsection{Atomic force microscopy (AFM) and piezo force microscopy (PFM)}

The morphological/piezoelectric characterization of $\mathrm{P}(\mathrm{VDF}-\mathrm{co}-\mathrm{TrFE})$ fibers deposited onto indium tin oxide (ITO)-coated glass substrates at a collector speed of $90 \mathrm{~mm} / \mathrm{min}$ and temperature of $120^{\circ} \mathrm{C}$ was carried out by atomic force microscopy (AFM) and piezo-force microscopy PFM. Measurements were done in contact mode by using a conductive diamondcoated probe with a nominal spring constant of $80 \mathrm{~N} \mathrm{~m}^{-1}$ (Bruker, USA) on a Bruker Dimension Icon system, equipped with a Nanoscope V controller. The surface roughness was calculated as the average of the root mean square values for areas of $25 \mu \mathrm{m}^{2}$ distributed along the fiber backbone. 


\section{Results}

\subsection{Fiber fabrication}

The processability of the $\mathrm{P}(\mathrm{VDF}-\mathrm{co}-\mathrm{TrFE})$ via $\mathrm{MEW}$ was initially tested using parameters traditionally used for this technology, however extruding $\mathrm{P}(\mathrm{VDF}-\mathrm{co}-\mathrm{TrFE})$ proved to be difficult compared to other polymers. Owing to the high viscosity of polymer (MFI = $25 \mathrm{~g} / 10$ min, ASTM D1238), a pressure greater than 1 bar was tested for polymer extrusion and jet initiation. However, it was observed that the pressurized gas would sometimes push through within the nozzle, resulting in poor extrusion. Following jet stabilization at high pressures, the oscillating jet (Supporting Video S1, 0.25x original speed) became electrostatically attracted towards the print head and lead to jet breakup (Supporting Video S2, 1.5x original speed). Therefore, despite a high viscosity of the $\mathrm{P}(\mathrm{VDF}-\mathrm{co}-\mathrm{TrFE})$, a lower $\mathrm{N}_{2}$ pressure of $0.5 \pm 0.1$ bar was selected for further experiments.

Further experiments revealed that due to the rapid solidification of the polymer melt, the extruded material did not adhere to the collector and was dragged over the surface of the collector (Supporting Video S3), similar as previously observed for polypropylene (PP) [23]. This issue of jet dragging was solved using collector heated to temperatures of $120-135^{\circ} \mathrm{C}$. P(VDF-co-TrFE) fibers were printed onto glass slides using two different collector temperatures. The resulting fibers were uniform and demonstrated good adherence to the collector substrate with the possibility to stack up to 20 alternating layers in $0^{\circ}-90^{\circ}$ orientation. Using SEM imaging for fiber morphology, it was revealed that fibrils are formed normal to the fiber orientation during the crystallization and solidification process of the polymer melt (Figure 1). Similar results, showing extended grain growth and crystallization for solution electrospun fibers at annealing temperatures above $115^{\circ} \mathrm{C}$, were observed by Kim et al. [24]. Melt electrospun polymers have also shown similar surface morphologies [25]. 

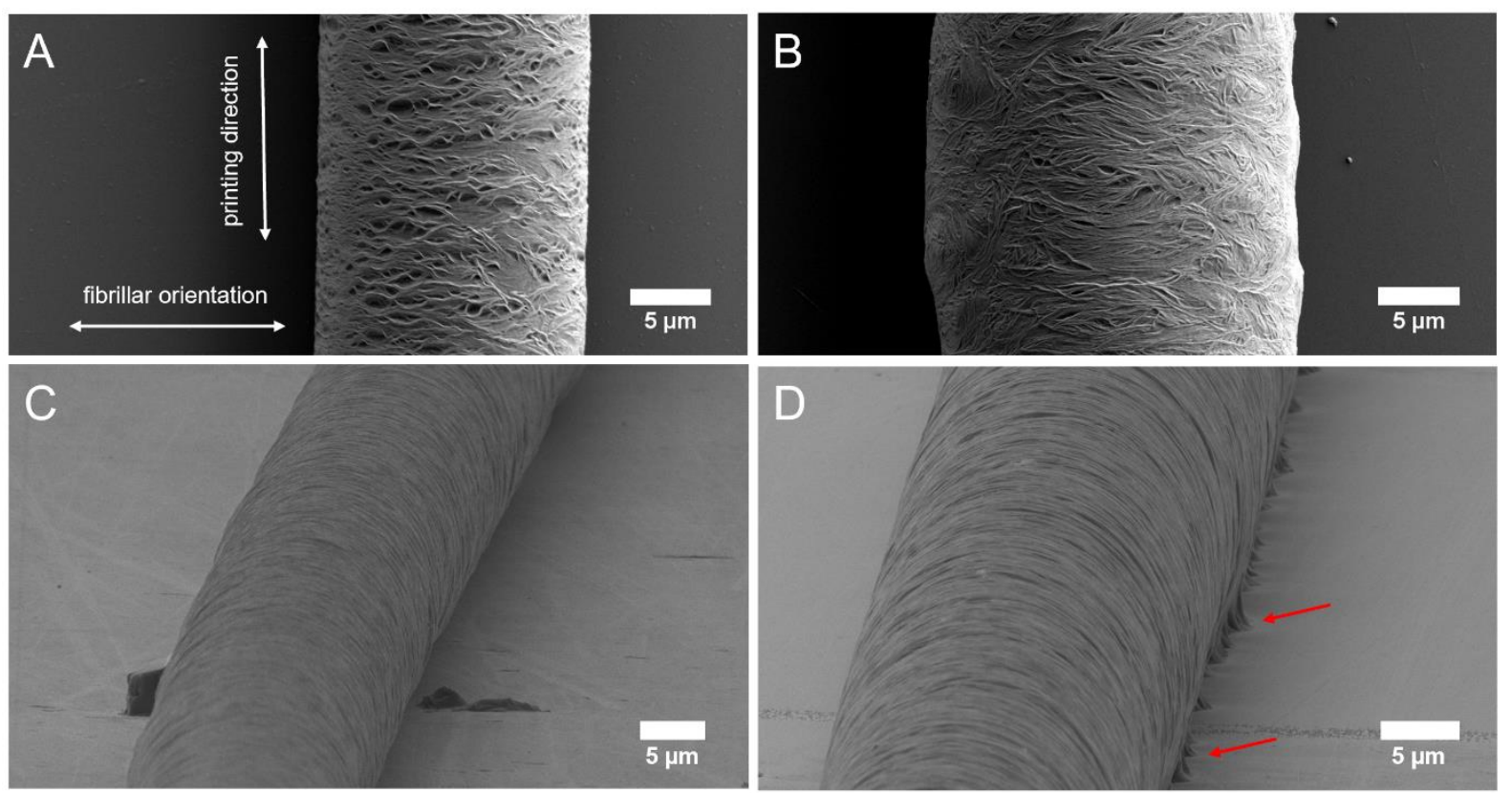

Figure 1. SEM images of fibers direct-written at $50 \mathrm{~mm} / \mathrm{min}$ onto collectors heated at A, C) $120^{\circ} \mathrm{C}$ and $\mathrm{B}, \mathrm{D}) 135^{\circ} \mathrm{C}$. SEM images showing the A-B) top-view of fibers depicting the printing direction and fibrillar orientation and C-D) showing side-view of the fibers. Fiber fusion and melting on the heated collector is highlighted using red arrows (D).

Using a collector temperature of $135^{\circ} \mathrm{C}$, the fibers start to show partial fusion and flattening onto the collector surface, consequently losing its circular shape (Figure 1D). Removing the printed fibers and scaffolds from the glass slides without damage was challenging for the higher collector temperature of $135^{\circ} \mathrm{C}$, as the scaffolds adhered more to the glass surface. A lower collector temperature of $120^{\circ} \mathrm{C}$ results in uniform and stable fibers that can be processed without noticeable flattening and could be detached without difficulties. Therefore, the use of a heated collector adds another adjustable instrument parameter for MEW of polymers where jet initiation is an issue due to non-adherence (Supporting Video S3).

One of the notable outcomes of this study, in strong contrast to all other polymers processed to date using MEW, is the very low collector speed required for direct-writing. Straight fibers, albeit with a larger $50 \mu \mathrm{m}$ diameter could be collected using collector speeds as low as $10 \mathrm{~mm} / \mathrm{min}$ and it was difficult to determine a critical translation speed (CTS), where a 
transition between sinusoidal and linear deposition indicates the speed of the electrified jet [21, 26]. Much higher CTS values were previously reported for other polymers including poly( $\varepsilon-$ caprolactone) $(300-750 \mathrm{~mm} / \mathrm{min})$ [21], urea-siloxane thermoplastic elastomers $(1500-3500$ $\mathrm{mm} / \mathrm{min})$ [19], and poly( $\varepsilon$-caprolactone-co-acryloyl carbonate) $(150-750 \mathrm{~mm} / \mathrm{min})$ [17]. The lowest CTS value previously reported was for PP $(50-150 \mathrm{~mm} / \mathrm{min})$, which is also a polymer that required a heated collector for direct writing [23]. This indicates that rapid cooling of the jet is likely responsible for the lack of a CTS in this instance. It was observed that above a maximum collector speed of approximately $100 \mathrm{~mm} / \mathrm{min}$ the molten jet readily "snapped" and continuous direct writing could not be achieved. Clearly, MEW processing parameters for $\mathrm{P}(\mathrm{VDF}-\mathrm{co}-\mathrm{TrFE})$ differ rather significantly from all previously processed materials.

Thermal analysis of raw polymer and scaffolds was performed to study the effect of high processing temperatures on $\mathrm{P}(\mathrm{VDF}-\mathrm{co}-\mathrm{TrFE})$. TGA revealed that the mass of the $\mathrm{P}(\mathrm{VDF}-\mathrm{co}-$ TrFE) melt remains constant over the measured period of $5 \mathrm{~h}$ at $170^{\circ} \mathrm{C}$ to simulate the conditions during the MEW process (Figure S1A). A color change of the melt from colorless/transparent to yellow-brown could be observed in line with that similarly observed for PVDF [22]. This color change may be attributed to low molar mass additives or unknown residues from the synthesis, rather than the polymer. At higher temperatures around $300^{\circ} \mathrm{C}$, TGA shows a significant reduction in the mass representing the onset of degradation of polymer. In the DSC measurements (Figure S1B) both, the polymer and the scaffolds showed same peaks in the second heating cycle suggesting no change in the polymer due to MEW processing. The cooling curves from DSC (Figure S1B) showed a Curie transition approximately around $75-80^{\circ} \mathrm{C}$ for both powder and MEW scaffolds. This Curie transition is lower for the cooling of the $\mathrm{P}(\mathrm{VDF}-\mathrm{co}-\mathrm{TrFE})$, compared to the Curie transition measured upon heating $\left(135^{\circ} \mathrm{C}\right)$ of the polymer. 


\subsection{Effect of Collector Speed on Fiber diameter}

To investigate the influence of the collector speed on the crystallization process and fiber diameter, it was varied from $10-100 \mathrm{~mm} / \mathrm{min}$ in $10 \mathrm{~mm} / \mathrm{min}$ increments at the aforementioned collector temperatures of $120 \pm 5^{\circ} \mathrm{C}$ and $135 \pm 5^{\circ} \mathrm{C}$. Resulting fibers were imaged by SEM (Figure 2). Increasing the collector speed results in a decrease of the fiber diameter for both collector temperatures. Above $30 \mathrm{~mm} / \mathrm{min}$, the decrease in fiber diameter levels off at around $15 \mu \mathrm{m}$ for both collector temperatures (Figure S2A). A similar behavior has been described previously for PCL [27].

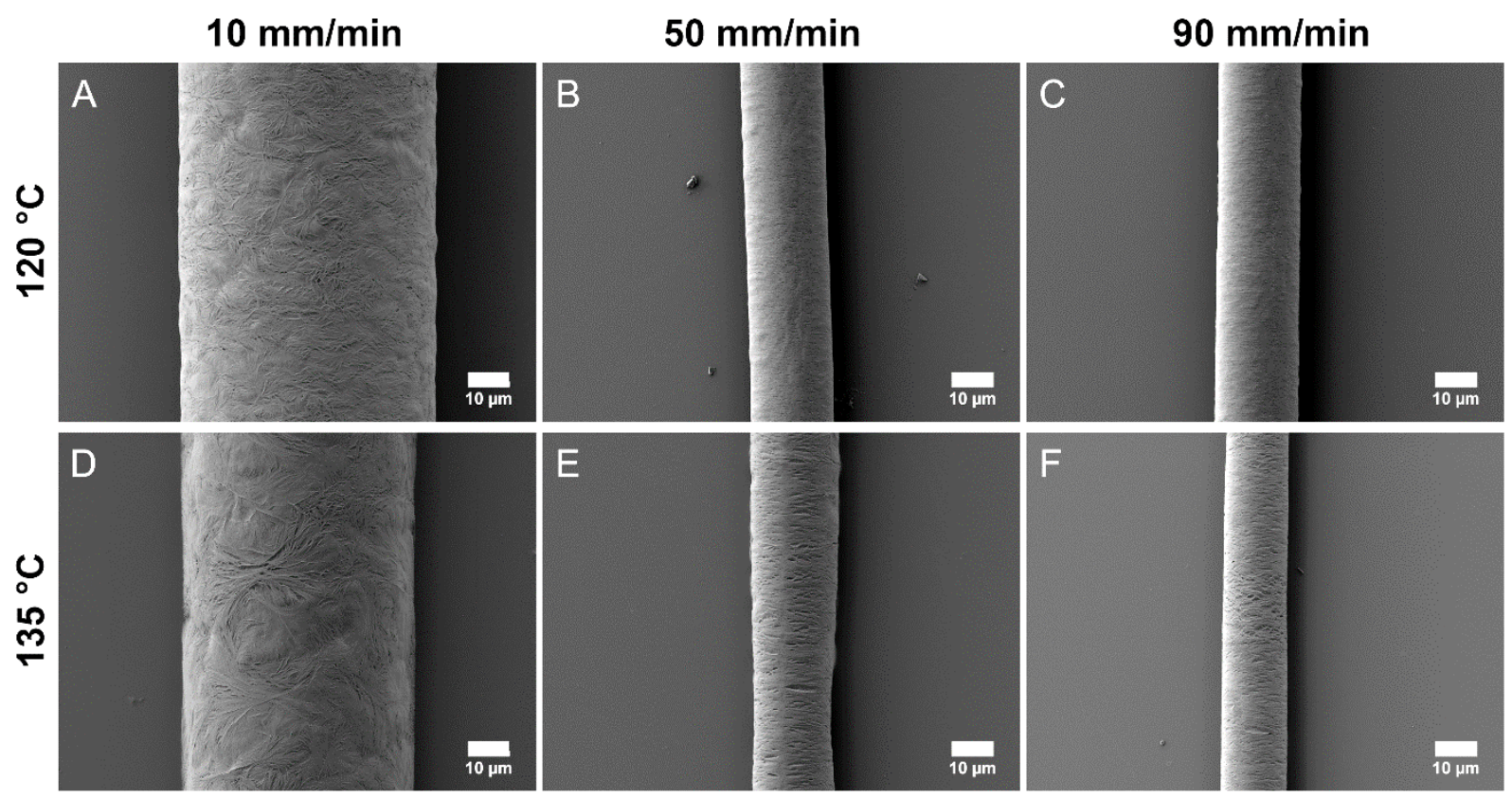

Figure 2. A-F) SEM images of MEW processed fibers printed at varying speeds $(10,50$ and $90 \mathrm{~mm} / \mathrm{min})$ and two different collector temperatures $\left(120^{\circ} \mathrm{C}\right.$ and $\left.135^{\circ} \mathrm{C}\right)$.

SEM images also showed differences in formation of crystalline domains with varying fiber diameters. The change in the fiber morphology depending on the collector speed can be attributed to changes in collector temperature and cooling behavior of the jet. A change in the fiber morphology depending on the annealing temperature was reported in the literature [24]. The fiber morphology can be related to grain growth and crystallization [24] and might be 
explained by a phase transition from the $\alpha$-phase into the piezoelectric, all-trans zig-zag $\beta$-phase. This phase transformation was previously described by different groups [28-30] as a transformation from a spherulitic to a microfibrillar structure and can be induced by stretching of the molten jet. The radial spherulitic structure is visible on MEW fibers direct-written with a collector speed of $10 \mathrm{~mm} / \mathrm{min}$ (Figure $\mathbf{2 A}$ and D) and transforms into different surface morphologies with increasing collector speed (Figure 2B-C, E-F and magnified view in Figure 1). Those surface morphologies could be caused by the stretching of the jet into a fibrillar structure from crystallites $[28,30]$. Furthermore, the collector speed influenced the jet lag of the polymer melt $[21,31]$ (Supporting Video S4, speed of the videos was adjusted to match the print duration of $10 \mathrm{~mm} / \mathrm{min}$ prints) and might lead to different cooling rates of the printed fibers, which in turn influences the crystallinity of the fibers. As previously observed for MEWprocessed fibers, changes in the surface morphology can also originate from disparities in the solidification rates of the fiber surface and core [32]. It is known, that with increasing collector speeds, the lag increases stretching of the jet [21, 31].

\subsection{Layer stacking behavior}

With the direct-writing parameters for single fibers established, the fabrication of multilayered $0-90^{\circ}$ scaffolds, at $500 \mu \mathrm{m}$ hatch spacing, with up to ten alternating layers was investigated. Notably, and never reported before for MEW, printing more than three stacked layers on top of each other resulted in the formation of sinusoidal structures in-between the crossing points, when cooling down the collector to room temperature after printing (Figure 3). This phenomenon was studied for the two different collector temperatures, different collector speeds and scaffolds with $0-90^{\circ}$ and $0-45^{\circ}$ fiber orientation. The collector temperature while printing seems not to have any significant influence on the formation of sinusoidal fibers; only the collector temperature while cooling appears to impact this phenomenon (Supporting Video S5). 

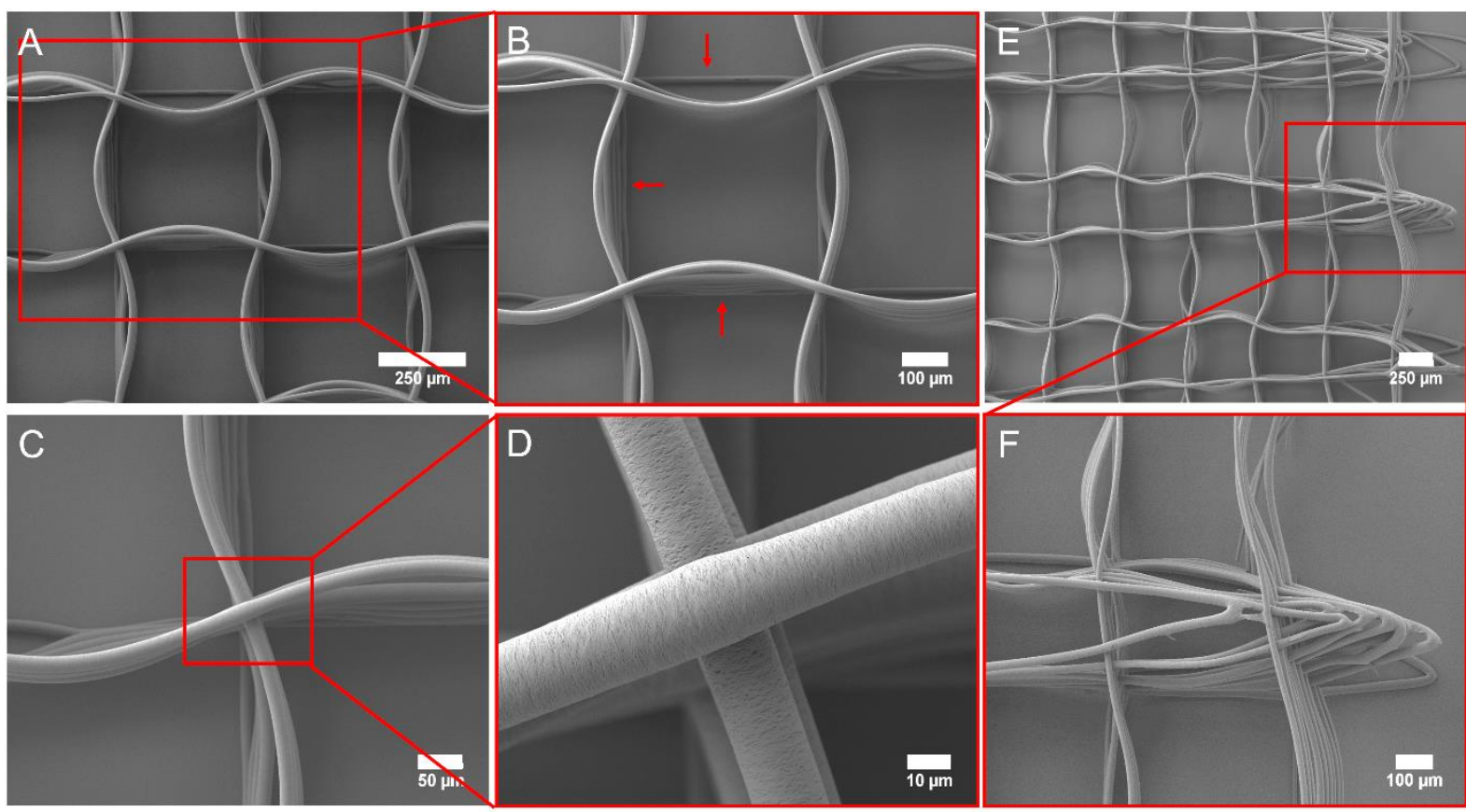

Figure 3. Collection of SEM images of a 0/90 ${ }^{\circ}$ scaffold. A-B) showing the box structure. Red arrows indicate the first three fibers deposited, which remain straight unlike the sinusoidal nature of the higher layers. SEM images of C, D) the crossing points and E-F) the turns. B, C and F) showing magnified views, respectively.

The sinusoidal formation starts to appear when the collector temperature reaches about 70$80^{\circ} \mathrm{C}$ at the glass slide and $80-90^{\circ} \mathrm{C}$ for the set-temperature of the heated collector (Supporting Video S5) and therefore could be connected to the Curie transition temperature during cooling as judged from the DSC thermograms (Figure S1B). These structural variations could be due to changes in the recrystallization process during solidification and residual thermal strain, which are known to be highly dependent on the cooling rate [32]. However, the first two to three layers always stay straight independent on the collector speed or the collector temperature, likely due to increased adherence to the collector and to the underlying layers (red arrows, Figure 3B).

When measuring the fiber diameter of the top and bottom layers of the scaffold (Figure S2B), no significant decrease or increase was observed. When inspecting the turns of the lines within the scaffolds (Figure 3E-F), the jet lag is pulling the fibers at the turns inwards, 
as previously observed for radial structures printed using medical-grade PCL [33]. Therefore, the position of the fiber changes with the number of layers when the directing of writing is substantially changing.

Furthermore, fabricated $\mathrm{P}(\mathrm{VDF}-\mathrm{co}-\mathrm{TrFE})$ scaffolds with 20 alternating layers in $0^{\circ}$ and $90^{\circ}$ showed the solidified fiber breaking in-between the scaffolds at various locations. The breakage of the fiber could be due to 1) the jet breaking or 2) mechanical forces associated with fiber bending while cooling. Figures 4A-B show an overview of a 20-layer P(VDF-co-TrFE) scaffold printed with a speed of $70 \mathrm{~mm} / \mathrm{min}$ and a collector temperature of $120^{\circ} \mathrm{C}$. At various locations one or more fibers are snapped, indicated with red arrows (Figure 4A-B), however a clear trend in where and when the fibers were breaking could not be determined. It seems that the fibers break likely due to rapid temperature changes, for example when removing the glass slides from the heated collector temperature $\left(120-135^{\circ} \mathrm{C}\right)$ to a surface at room temperature.
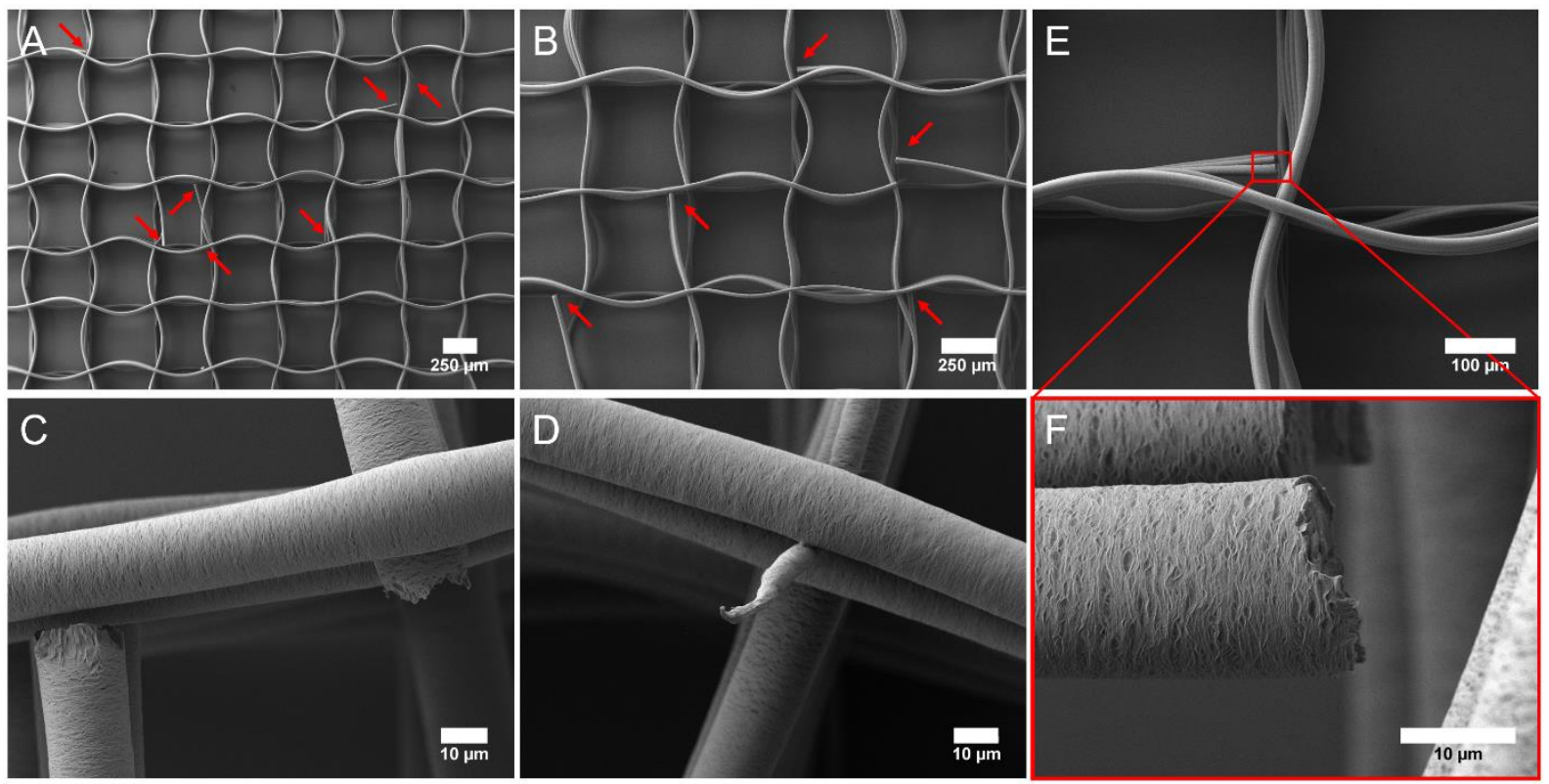

Figure 4. SEM images of 20 alternating layers with broken fibers and fiber bundle. A-B) box structured scaffold with red arrows indicating brittle fractures. Magnified SEM images highlighting the differences between C) a brittle fracture due to the bending/cooling crystallization process and D) a snapped fiber caused by jet break-up. E) showing sharp breaks resulting in fiber bundles with F) as a magnified view. 
Another indication of fibers breaking post solidification is that the broken fiber bundles remain at their position within the scaffold. In comparison, a jet break-up in the polymer melt, the point of break is stretched and pulled until the jet snaps, resembling ductile failure behavior (Figure 4D).

\subsection{XRD measurements, crystallinity and $\beta$-phase content}

The corresponding XRD measurements for the raw powder (Figure S3A and S4A) and the scaffolds printed at collector temperatures of $120^{\circ} \mathrm{C}$ (Figure S3B and S4B) and $135^{\circ} \mathrm{C}$ (Figure S3C and S4C) are summarized in Figure S4. For the unprocessed P(VDF-co-TrFE) powder, a clearly visible shoulder peak can be seen at $18^{\circ}$ and a peak at $20^{\circ}$, respectively, which can be attributed to the polar $\beta$-phase (110 and 200), as described previously for SES fibers [4, 24]. For all MEW-processed scaffolds the shoulder-peak at $18^{\circ}$, attributed to the $\alpha$-phase, disappeared or is reduced significantly. Comparing the measurements in Figure S4B, the peak at $20^{\circ}$ is broader for the scaffolds printed with $50 \mathrm{~mm} / \mathrm{min}$ and $70 \mathrm{~mm} / \mathrm{min}$ (Figure S3). A similar broader peak can be seen at the collector temperature of $135^{\circ} \mathrm{C}$ for the scaffold printed with $100 \mathrm{~mm} / \mathrm{min}$. Therefore, MEW processing the raw powder seems to decrease the $\alpha$-phase, as previously observed for SES fibers due to the in situ poling and stretching while electrospinning $[4,12,24]$.

From the XRD results, the degree of crystallinity for the MEW-processed scaffolds printed at both collector temperatures can be calculated (Table 1 and Figure S3). For the scaffolds printed at $120^{\circ} \mathrm{C}$, the total crystallinity is similar to the crystallinity of the unprocessed powder and significantly higher compared to the scaffolds printed at $135^{\circ} \mathrm{C}$. For both collector temperatures, the ratio between $\alpha$ - and $\beta$-phase content did change with $\beta$-phase contents at around $80 \%$ of the total crystallinity for the MEW-processed scaffolds. Therefore, MEWprocessing the $\mathrm{P}(\mathrm{VDF}-\mathrm{co}-\mathrm{TrFE})$ with a heated collector could be seen as an in situ annealing 
process and annealing temperatures close to the Curie temperature can provide sufficient energy for chain reorientation and, therefore, can lead to an increase in $\beta$-phase formation compared to unprocessed material [24].

For the samples printed at the higher collector temperature with speeds of $50 \mathrm{~mm} / \mathrm{min}$ and $70 \mathrm{~mm} / \mathrm{min}$ the $\beta$-phase content is around $85 \%$, however, the total crystallinity is significantly less compared to the samples printed at a collector temperature of $120^{\circ} \mathrm{C}$ (Table 1).

Table 1. Calculated total crystallinity and related $\alpha-, \beta$-phase contents of the total crystallinity for the MEW-processed scaffolds printed at 50,70 and $100 \mathrm{~mm} / \mathrm{min}$ at collector temperatures of $120^{\circ} \mathrm{C}$ and $135^{\circ} \mathrm{C}$, respectively compared to the unprocessed powder.

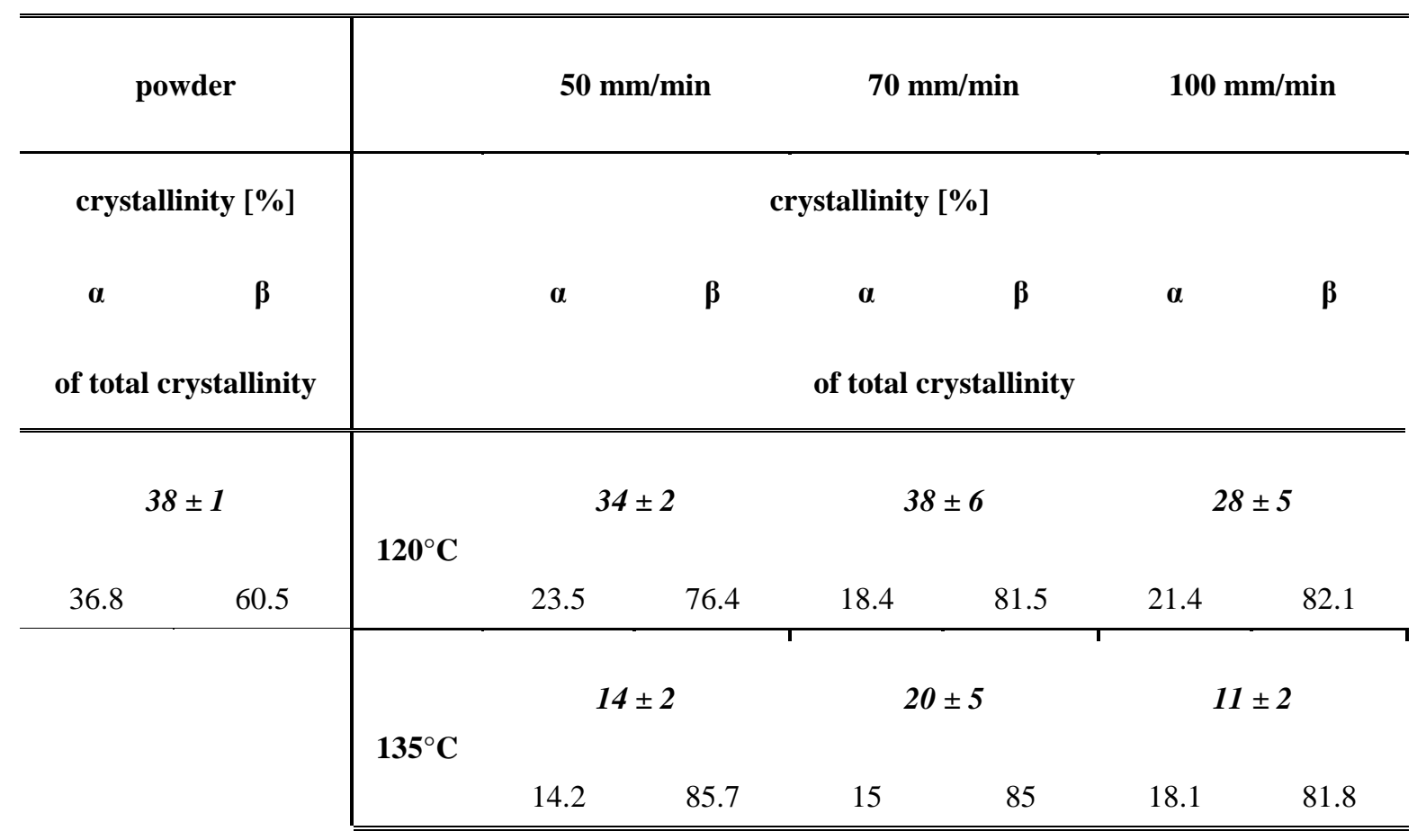

The total crystallinity of the samples printed at a collector temperature of $135^{\circ} \mathrm{C}$ is between $11 \pm 2 \%$ and $20 \pm 45 \%$, compared to $28 \pm 5 \%$ and $38 \pm 6 \%$ for the collector temperature of $120^{\circ} \mathrm{C}$. Using the set temperature and the actual measured collector temperatures, we were able to quantify the cooling rates with a slightly faster cooling rate for the $135^{\circ} \mathrm{C}$ collector temperature. Therefore, the collector with the set temperature of $135^{\circ} \mathrm{C}$ might lead to a decrease 
in crystallinity, as it is known that the crystallinity is highly dependent on the cooling rate and a fast cooling rate can lead to a lower crystallinity $[34,35]$.

\subsection{Jet behavior}

Rapid cooling of the polymer was believed to be the reason for dragging of the polymer jet on the collector as discussed above. This issue was simply circumvented by using a heated collector. Another interesting phenomenon shown for the copolymer jet is the oscillating/vibrating behavior as depicted in Supporting Video S1. The balance between the amount of polymer and voltage applied was found to be the key to laydown fibers consistently for long durations. As the pressure was increased, the jet was readily attracted towards the head (Supporting Video S2) and disrupted the jetting process. The collector temperature of $120^{\circ} \mathrm{C}$ and the applied voltage was not sufficient enough to attract the jet and initiate the process of MEW.

The formation of a stable jet while printing is highly dependent on the processing parameters, especially the speed of the collector [21, 36-38]. For other already established MEW-processable polymers, the CTS is a very important value and is sensitive to small changes in the processing parameters. The jet at speeds just above the CTS is typically starting with a nearly vertical line and then increasing the lag significantly until the lag seems to level off [38], which can be related to the fiber diameter discussed previously. For the P(VDF-coTrFE) a similar behavior was observed, however, no CTS was found and the processed fibers resulted in straight and uniform lines even at significant low speeds of $10 \mathrm{~mm} / \mathrm{min}$. Looking at the jet lag of the $\mathrm{P}(\mathrm{VDF}-\mathrm{co}-\mathrm{TrFE})$, within the processable speed range from 10 to $100 \mathrm{~mm} / \mathrm{min}$, an increase in the jet lag depending on the collector speed was detected and the jet lag seems to be leveled off at $50 \mathrm{~mm} / \mathrm{min}$ and higher (Supporting Video S4). 


\subsection{Morphological and piezoelectric characterization}

The piezoelectric response of the MEW-processed fiber was measured by PFM upon the application of an alternated (AC) voltage with frequency of $15 \mathrm{kHz}$ (Figure 5). The measured fiber was deposited onto an ITO-coated glass substrate at a collector speed of $90 \mathrm{~mm} / \mathrm{min}$ and a temperature of $120^{\circ} \mathrm{C}$. The fiber diameter, taking into account a deconvolution of the profile of the used AFM tip, is $16 \mu \mathrm{m}$ and the surface roughness along the fiber backbone is of the order of $100 \mathrm{~nm}$. Upon the application of an AC bias of $10 \mathrm{~V}$ between the tip and the sample, the PFM amplitude signal indicates a strain response of tens of pm, which is higher along the fiber backbone (Figure 5C and F) highlighting a surface pattern that can be correlated with a local enhancement of the vertical component of the polarization vector in certain crystalline domains.

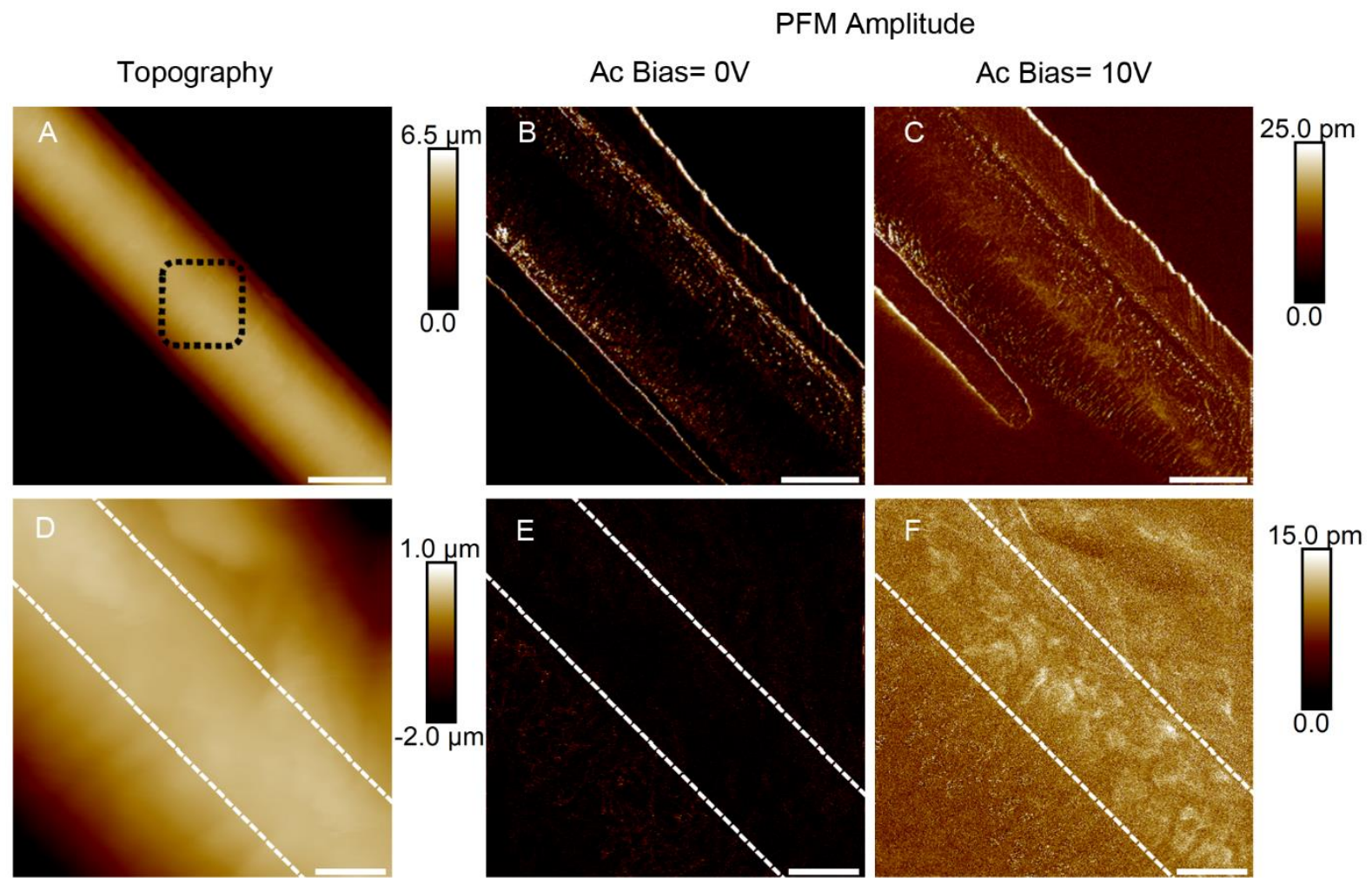

Figure 5. A) AFM topographic images and corresponding PFM images of a P(VDF-co-TrFE) fiber at AC bias voltage of B) $0 \mathrm{~V}$ and C) $10 \mathrm{~V}$. A-C) Scale bars: $10 \mu \mathrm{m}$. D-F) are high-magnification images of A-C) measured in the area of the fiber highlighted by the black box in A). The dashed white lines highlight the fiber backbone. D-F) Scale bars: $2 \mu \mathrm{m}$. 
On the contrary, the amplitude of the in-plane oscillation, that was measured by lateral PFM (LPFM) indicates an almost uniform distribution of the in-plane piezo response (Figure S5) suggesting different dynamics of crystallization and solidification along the fiber backbone. Changes in the PFM amplitude signal were measured for AC bias in the interval 2-10 V, leading to a linear dependence between the piezo response and the applied voltage as typical of piezoelectricity (Figure S6A). Dipoles are sensitive to the direction of the applied field and exhibit a phase shift when the external bias is inverted (i.e. the positive bias is applied to the sample and the tip is grounded) as reported in Figure S6 B-C.

\section{Conclusion}

In this study, an important piezoelectric polymer, $\mathrm{P}(\mathrm{VDF}-\mathrm{co}-\mathrm{TrFE})$, was shown to be processable via MEW into fibers ranging between $10 \mu \mathrm{m}$ and $50 \mu \mathrm{m}$, depending on the collector speed. The direct-writing had to be performed at remarkably low speeds of less than $100 \mathrm{~mm} / \mathrm{min}$ while a heated collector was essential to initiate the direct writing. Upon cooling of the collector, straight fibers formed sinusoidal structures, which was correlated to a change in the macromolecular orientation within the recrystallization process, in turn influenced by the collector temperature and speed. The electroactive nature of this polymer was proven by PFM measurements and makes this study relevant to applications where well-resolved 3D printed structures of such piezoelectric polymers are of interest, such as biomedical products, energy harvesting, actuators and sensors.

\section{Acknowledgements}

We gratefully acknowledge financial support by the Volkswagen Foundation (grant number 93418) and additionally J. C. K. is supported by the Joachim Herz Foundation. The technical assistance of Philipp Stahlhut and Judith Friedlein for SEM imaging is appreciated while the Zeiss Crossbeam CB 340 SEM was funded by the German Research Foundation (DFG) State 
Major Instrumentation Programme (INST 105022/58-1 FUGG). Furthermore, we thank Dr. Tomasz Jüngst for providing the camera and Michael Bartolf-Kopp for his input and help for the supporting videos. Proofreading by Prof. Dr. Uwe Gbureck is greatly appreciated. 


\section{References}

[1] A.H. Rajabi, M. Jaffe, T.L. Arinzeh, Piezoelectric materials for tissue regeneration: A review, Acta Biomaterialia 24 (2015) 12-23.

[2] A. Zaszczynska, P. Sajkiewicz, A. Gradys, Piezoelectric Scaffolds as Smart Materials for Neural Tissue Engineering, Polymers 12(1) (2020).

[3] X. Liu, X. Wang, H. Zhao, Y. Du, Myocardial Cell Pattern on Piezoelectric Nanofiber Mats for Energy Harvesting, Journal of Physics: Conference Series 557 (2014) 012057.

[4] L. Persano, C. Dagdeviren, Y. Su, Y. Zhang, S. Girardo, D. Pisignano, Y. Huang, J.A. Rogers, High performance piezoelectric devices based on aligned arrays of nanofibers of poly(vinylidenefluoride-cotrifluoroethylene), Nature Communications 4(1) (2013) 1633.

[5] S. Wu, M.-S. Chen, P. Maurel, Y.-s. Lee, M.B. Bunge, T.L. Arinzeh, Aligned fibrous PVDF-TrFE scaffolds with Schwann cells support neurite extension and myelination in vitro, Journal of Neural Engineering 15(5) (2018) 056010.

[6] B. Zaarour, L. Zhu, C. Huang, X. Jin, Enhanced piezoelectric properties of randomly oriented and aligned electrospun PVDF fibers by regulating the surface morphology, Journal of Applied Polymer Science 136(6) (2019) 47049.

[7] F.S. Foster, K.A. Harasiewicz, M.D. Sherar, A history of medical and biological imaging with polyvinylidene fluoride (PVDF) transducers, IEEE Transactions on Ultrasonics, Ferroelectrics, and Frequency Control 47(6) (2000) 1363-1371.

[8] P. Martins, A.C. Lopes, S. Lanceros-Mendez, Electroactive phases of poly(vinylidene fluoride): Determination, processing and applications, Progress in Polymer Science 39(4) (2014) 683-706.

[9] R.I. Haque, R. Vié, M. Germainy, L. Valbin, P. Benaben, X. Boddaert, Inkjet printing of high molecular weight PVDF-TrFE for flexible electronics, Flexible and Printed Electronics 1(1) (2015) 015001.

[10] C. Wan, C.R. Bowen, Multiscale-structuring of polyvinylidene fluoride for energy harvesting: the impact of molecular-, micro- and macro-structure, Journal of Materials Chemistry A 5(7) (2017) 30913128.

[11] J. Hafner, M. Teuschel, M. Schneider, U. Schmid, Origin of the strong temperature effect on the piezoelectric response of the ferroelectric (co-)polymer P(VDF70-TrFE30), Polymer 170 (2019) 1-6.

[12] S.M. Damaraju, Y. Shen, E. Elele, B. Khusid, A. Eshghinejad, J. Li, M. Jaffe, T.L. Arinzeh, Threedimensional piezoelectric fibrous scaffolds selectively promote mesenchymal stem cell differentiation, Biomaterials 149 (2017) 51-62.

[13] S.M. Damaraju, S. Wu, M. Jaffe, T.L. Arinzeh, Structural changes in PVDF fibers due to electrospinning and its effect on biological function, Biomedical Materials 8(4) (2013) 045007.

[14] Y.-S. Lee, T.L. Arinzeh, The Influence of Piezoelectric Scaffolds on Neural Differentiation of Human Neural Stem/Progenitor Cells, Tissue Engineering Part A 18(19-20) (2012) 2063-2072.

[15] Y.-S. Lee, G. Collins, T. Livingston Arinzeh, Neurite extension of primary neurons on electrospun piezoelectric scaffolds, Acta Biomaterialia 7(11) (2011) 3877-3886.

[16] N. Weber, Y.S. Lee, S. Shanmugasundaram, M. Jaffe, T.L. Arinzeh, Characterization and in vitro cytocompatibility of piezoelectric electrospun scaffolds, Acta Biomaterialia 6(9) (2010) 3550-3556.

[17] G. Hochleitner, F. Chen, C. Blum, P.D. Dalton, B. Amsden, J. Groll, Melt electrowriting below the critical translation speed to fabricate crimped elastomer scaffolds with non-linear extension behaviour mimicking that of ligaments and tendons, Acta Biomaterialia 72 (2018) 110-120.

[18] M. Castilho, A. van Mil, M. Maher, C.H.G. Metz, G. Hochleitner, J. Groll, P.A. Doevendans, K. Ito, J.P.G. Sluijter, J. Malda, Melt Electrowriting Allows Tailored Microstructural and Mechanical Design of Scaffolds to Advance Functional Human Myocardial Tissue Formation, Advanced Functional Materials 28(40) (2018) 1803151.

[19] G. Hochleitner, E. Fürsattel, R. Giesa, J. Groll, H.-W. Schmidt, P.D. Dalton, Melt Electrowriting of Thermoplastic Elastomers, Macromolecular Rapid Communications 39(10) (2018) 1800055.

[20] G. Hochleitner, T. Jüngst, T.D. Brown, K. Hahn, C. Moseke, F. Jakob, P.D. Dalton, J. Groll, Additive manufacturing of scaffolds with sub-micron filaments via melt electrospinning writing, Biofabrication 7(3) (2015) 035002. 
[21] A. Hrynevich, B.S. Elci, J.N. Haigh, R. McMaster, A. Youssef, C. Blum, T. Blunk, G. Hochleitner, J. Groll, P.D. Dalton, Dimension-Based Design of Melt Electrowritten Scaffolds, Small 14(22) (2018) e1800232.

[22] S. Florczak, T. Lorson, T. Zheng, M. Mrlik, D.W. Hutmacher, M.J. Higgins, R. Luxenhofer, P.D. Dalton, Melt electrowriting of electroactive poly(vinylidene difluoride) fibers, Polymer International 68(4) (2019) 735-745.

[23] J.N. Haigh, T.R. Dargaville, P.D. Dalton, Additive manufacturing with polypropylene microfibers, Materials Science and Engineering: C 77 (2017) 883-887.

[24] M. Kim, S. Lee, Y.-i. Kim, Solvent-controlled crystalline beta-phase formation in electrospun P(VDFTrFE) fibers for enhanced piezoelectric energy harvesting, APL Materials 8(7) (2020) 071109.

[25] P.D. Dalton, D. Grafahrend, K. Klinkhammer, D. Klee, M. Moller, Electrospinning of polymer melts: phenomenological observations, Polymer 48(23) (2007) 6823-6833.

[26] P.D. Dalton, Melt electrowriting with additive manufacturing principles, Current Opinion in Biomedical Engineering 2 (2017) 49-57.

[27] T.D. Brown, P.D. Dalton, D.W. Hutmacher, Direct Writing By Way of Melt Electrospinning, Advanced Materials 23(47) (2011) 5651-5657.

[28] J. Gomes, J. Serrado Nunes, V. Sencadas, S. Lanceros-Mendez, Influence of the $\beta$-phase content and degree of crystallinity on the piezo- and ferroelectric properties of poly(vinylidene fluoride), Smart Materials and Structures 19(6) (2010) 065010.

[29] J.B. Lando, W.W. Doll, The polymorphism of poly(vinylidene fluoride). I. The effect of head-tohead structure, Journal of Macromolecular Science, Part B 2(2) (1968) 205-218.

[30] V. Sencadas, R. Gregorio, S. Lanceros-Méndez, $\alpha$ to $\beta$ Phase Transformation and Microestructural Changes of PVDF Films Induced by Uniaxial Stretch, Journal of Macromolecular Science, Part B 48(3) (2009) 514-525.

[31] I. Liashenko, A. Hrynevich, P.D. Dalton, Designing Outside the Box: Unlocking the Geometric Freedom of Melt Electrowriting using Microscale Layer Shifting, Advanced Materials n/a(n/a) (2020) 2001874.

[32] J. Uribe-Gomez, A. Posada-Murcia, A. Shukla, M. Ergin, G. Constante, I. Apsite, D. Martin, M. Schwarzer, A. Caspari, A. Synytska, S. Salehi, L. Ionov, Shape-Morphing Fibrous Hydrogel/Elastomer Bilayers Fabricated by a Combination of 3D Printing and Melt Electrowriting for Muscle Tissue Regeneration, ACS Applied Bio Materials (2021).

[33] E. Bakirci, N. Schaefer, O. Dahri, A. Hrynevich, P. Strissel, R. Strick, P.D. Dalton, C. Villmann, Melt Electrowritten In Vitro Radial Device to Study Cell Growth and Migration, Advanced Biosystems $\mathrm{n} / \mathrm{a}(\mathrm{n} / \mathrm{a}) 2000077$.

[34] J.E.K. Schawe, Cooling rate dependence of the crystallinity at nonisothermal crystallization of polymers: A phenomenological model, Journal of Applied Polymer Science 133(6) (2016).

[35] C. Fischer, D. Drummer, Crystallization and Mechanical Properties of Polypropylene under Processing-Relevant Cooling Conditions with respect to Isothermal Holding Time, International Journal of Polymer Science 2016 (2016) 5450708.

[36] F.M. Wunner, P. Mieszczanek, O. Bas, S. Eggert, J. Maartens, P.D. Dalton, E.M. De-Juan-Pardo, D.W. Hutmacher, Printomics: the high-throughput analysis of printing parameters applied to melt electrowriting, Biofabrication 11(2) (2019) 025004.

[37] A. Hrynevich, B.Ş. Elçi, J.N. Haigh, R. McMaster, A. Youssef, C. Blum, T. Blunk, G. Hochleitner, J. Groll, P.D. Dalton, Dimension-Based Design of Melt Electrowritten Scaffolds, Small 14(22) (2018) 1800232.

[38] A. Hrynevich, I. Liashenko, P.D. Dalton, Accurate Prediction of Melt Electrowritten Laydown Patterns from Simple Geometrical Considerations, Advanced Materials Technologies n/a(n/a) 2000772. 